\title{
Fernerkundung als Mittel zur Bereitstellung von Planungsgrundlagen in der Dritten Welt: Das Beispiel Sri Lanka
}

\section{Das Projekt-Vorfeld}

\subsection{Zur Landbewirtschaftung in Sri Lanka}

Der tropische Inselstaat Sri Lanka vor der Südspitze Indiens ist mit seinen rund $65000 \mathrm{~km}^{2}$ etwa anderthalb mal so groß wie die Schweiz. Die Bevölkerung zählt heute rund 15 Millionen Menschen, die sich im Südwesten, ganz im Norden und entlang der Ostküste konzentrieren. Die Landgebiete im zentralen Norden und Osten bilden die Pionierzone mit vielen Neulandkolonisationen. Die ceylonesische Wirtschaft stützt sich seit über 2000 Jahren auf die Landwirtschaft ab, welche hochentwickelte Produktionsweisen der Subsistenz hervorgebracht hat: Tausende von kleineren und größeren Staubecken sammeln das Regenwasser der Monsune, um damit Naßreiskulturen zu ermöglichen. Ergänzt wird dieses System durch Nahrungsmittelproduktion auf Brandrodungsflächen im umliegenden Dschungelgebiet.

Die Besetzung Sri Lankas durch ausländische Kolonialmächte mit Beginn des 16. Jahrhunderts, vorab durch die Portugiesen, dann die Holländer und vor allem durch die Engländer ab etwa 1800, verursachte einen tiefgreifenden Wandel in der Landbewirtschaftungsstruktur. Große Flächen wurden für exportorientierte Plantagen wie Tee, Kautschuk, Kokospalmen und Gewürze beansprucht und die Infrastruktureinrichtungen auf diesen Sektor ausgerichtet. Dies führte zu einer eigentlichen Vernachlässigung der Eigenversorgung. Erst seit den dreißiger Jahren dieses Jahrhunderts wurde wieder vermehrte Aufmerksamkeit auf die bäuerliche Landwirtschaft gerichtet; so begann man mit der Renovation alter Bewässerungsstrukturen und dem Bau neuer Anlagen.

Die Landerschließung beschleunigte sich nach der Unabhängigkeit 1948 und nimmt auch heute einen wichtigen Stellenwert in der ceylonesischen Planung ein.

Die Situation der Landressourcen in Sri Lanka in den letzten 10 bis 20 Jahren ist gekennzeichnet von der in vielen Ländern der Dritten Welt bekannten Nutzungsproblematik, die aufgrund des beschleunigten Bevölkerungswachstums, der Krise der Exportwirtschaft, neueren Trends in Richtung Agrobusiness u.a. zu schwerwiegenden ökologischen Problemen und Konflikten um Landreserven führte.

\subsection{Die Rolle des Survey Departments im Datenbeschaffungsproze $\beta$}

Das schon 1800 gegründete Survey Department (vergleichbar der Schweizer Landestopographie) spielte bald eine wichtige Rolle in der Bereitstellung von Grundlagen für Planungs- und Ausführungsarbeiten im Bereich Landbewirtschaftung. So führte und führt das Amt die für Landbesiedlungsvorhaben notwendigen Vermessungen und Landaufnahmen durch, zu denen etwa topographische Aufnahmen, Vermessung von Staubecken und Dämmen, Kanälen, Straßen und die Vermessung von Parzellen für die Landzuteilung an Siedler gehören. In den dreißiger Jahren begannen Katasterarbeiten und die Verteilung von Staatsland an verschiedenste Nutzer. Um 1956 fand eine erste landesweite Luftbildbefliegung statt mit begleitender Einführung photogrammetrischer Auswertungsmethoden für die topographische Kartierung; eine erste landesweite Erhebung der Landnutzung wurde ebenfalls durchgeführt.

Die massiven Entwicklungsvorhaben des ceylonesischen Staates absorbierten jedoch bald den größten Teil der Kapazitäten des Survey Departments. Dies führte zu einer zwangsläufigen Vernachlässigung der systematischen und landesweiten Aufnahmen und Nachführungen, insbesondere der Landbesitz- und Bewirtschaftungsstrukturen, welche sich vor allem in den letzten Jahren enorm veränderten. Gerade in der heutigen Zeit mit all ihren ökologischen und ökonomischen Problemen sind die zuständigen sektoralen Planungsstellen auf aktuelle und genaue Grundlageninformationen angewiesen. Das so entstandene Daten-

S. D. F. C. Nanayakkara*, Surveyor General of Sri Lanka und sri lankischer Projekt-Koordinator;

Urs Geiser* , Schweizer Projektleiter 1978-82;

Martin Sommer*, Schweizer Projektleiter 1983-84;

Rainer Humbel*, Schweizer Berater;

Michel Gelbert*, Schweizer Berater;

Peter Schmid**, Technische Betreuung;

* Survey Department, P.O. Box 506, Colombo 5, Sri Lanka

* Geographisches Institut der Universität Zürich, Winterthurerstraße 190, 8057 Zürich 
defizit betrifft Bereiche der Agrar-, Forst- und Plantagenwirtschaft, der Fischerei, des Umweltschutzes und der Stadtplanung gleichermaßen.

Die unbefriedigende Situation veranlaßte das Survey Department Mitte der siebziger Jahre zum Beginn zweier neuer Programme:

- die Herstellung neuer topographischer Karten im Maßstab von 1:50000 (für ausgewählte Gebiete $1: 10000$ ) aufgrund einer Neubefliegung des Landes - der Aufbau einer Abteilung zur thematischen Kartierung mit Hilfe von Fernerkundungstechniken.

Die unter diesen Programmen aufzuarbeitenden räumlichen Daten sollen als Ergänzung zu den sozioökonomischen Informationen für ländliche Gebiete verstanden werden, welche in Sri Lanka vom gut ausgebauten "Agrarian Research and Training Institute» erhoben werden.

\subsection{Die Entstehung des "Sri Lanka - Swiss Remote Sensing Project»}

Zur thematischen Analyse der im Jahre 1956 geflogenen Luftaufnahmen wurde am Survey Department eine kleine Ressourcen-Interpretationsgruppe aufgebaut. Sie begann sich später auch mit den aufkommenden neuen Fernerkundungstechniken (vorab Satellitenbilder) $\mathrm{zu}$ befassen und beteiligte sich an ersten Informationsprogrammen. Die gewonnenen Erfahrungen bekräftigten den Wunsch des Survey Departments, den Bereich der thematischen Kartierung zu intensivieren und dadurch einen Beitrag zur Behebung des akuten Datenmangels im Planungssektor zu leisten.

Um den notwendigen Technologie-Transfer im Bereich der Methoden wie auch der Ausbildung eigenen Personals zu vollziehen, wandte sich das Survey Department an den ceylonesischen Staat, um im Rahmen der internationalen Entwicklungszusammenarbeit die Möglichkeit für ein konkretes Projekt zu prüfen. Die Projektanfrage gelangte an die Schweizerische Direktion für Entwicklungszusammenarbeit (DEH), welche die Konzeptionalisierung und Durchführung des Projektes an die Fernerkundungsabteilung des Geographischen Institutes der Universität Zürich (Abteilung von Prof. Dr. H. Haefner und Prof. Dr. K. I. Itten) in Regie übergab. Als primäre Projektziele wurden formuliert:

- Mithilfe beim Aufbau eines Fernerkundungszentrums in Sri Lanka

- Ausbildung lokaler Fachkräfte

- Erarbeitung geeigneter Methoden zur Durchführung von Datenerhebungen mittels Fernerkundungstechniken

- Durchführung konkreter Datenerhebungen (zu Beginn wurde Schwergewicht auf Reisproduktionsdaten gelegt).

\section{Zur Konzeption des Projektes}

\subsection{Die Strukturierung des Projektverlaufes}

Traditionelle Methoden der Luftbildinterpretation werden weltweit operationell eingesetzt. Neue Techniken der Satellitenbildanalyse befinden sich vor allem im Stadium der Entwicklung und Erprobung. Der Regieträger erachtete es deshalb als angezeigt, ein konkretes Projektprogramm erst nach vorangehenden Untersuchungen über die operationelle Durchführbarkeit der Projektziele zu formulieren.

Aufgrund dieser Einsicht wurde eine einjährige Pilotphase formuliert und 1978 durchgeführt. Zwei Schweizer Geographen unternahmen in Zusammenarbeit mit dem Survey Department vor Ort eingehende Untersuchungen in Testgebieten, um methodische Ansätze zu entwickeln. Die Phase ermöglichte eine gezielte Analyse der Fragestellung, ohne diese durch den Druck- und Zugzwang bereits operationeller Projektarbeiten zu belasten. Die gemachten Erfahrungen (SSRSP 1978) dienten zur Planung des eigentlichen Projektvorhabens (SSRSP 1979).

Die konkrete Projektumsetzung in Sri Lanka begann im Januar 1980, begleitet von drei Schweizern. In einer wiederum etwa einjährigen Testphase wurden die aufgrund der Pilotphase formulierten Methoden im Feldeinsatz geprüft und konkretisiert (SSRSP 1981). Die eigentliche Implementierungsphase begann somit im Frühjahr 1981 und ist gegenwärtig am Laufen.

In der Zweijahresphase 1985/86 soll die landesweite Landnutzungskartierung fertiggestellt werden. Neben der Festigung der technischen Abläufe soll dabei Hauptgewicht auf organisatorische Aspekte gelegt werden. Ziel dieser Phase ist die sukzessive Reduzierung des Schweizerischen Engagements und damit Übergabe des Projektes; zu diesem Zweck arbeiten ab Beginn 1985 noch zwei Schweizer am Zentrum.

Im folgenden sollen die konzeptionellen Ansätze vorgestellt werden, wie sie aufgrund der verschiedenen, von wichtigen Lernprozessen geprägten Phasen entstanden sind.

\subsection{Zum technischen Konzept}

Neuere Fernerkundungstechniken erlauben im Gegensatz zu konventionellen Luftaufnahmen eine bessere großflächige Bedeckung in kürzeren Zeitabständen. Dies gilt vor allem für Satellitenbilder des Typs Landsat (für eine Beschreibung siehe etwa HAEFNER 1975). Mit einem Multispektralscanner erfaßt der Satellit theoretisch alle 18 Tage das selbe Gebiet. Ein einzelnes Landsat-Bild bedeckt ein Gebiet von $185 \times 185 \mathrm{~km}$ (ca. $30000 \mathrm{~km}^{2}$ ) bei einer Bodenauflösung von etwa 80 Metern. Die für Sri Lanka benötigten Bilder sind in Abb. 1 gezeigt. Heute besteht ein Netz von Bodenempfangsstationen verteilt über die ganze Welt; im Falle Sri Lankas können somit Land- 


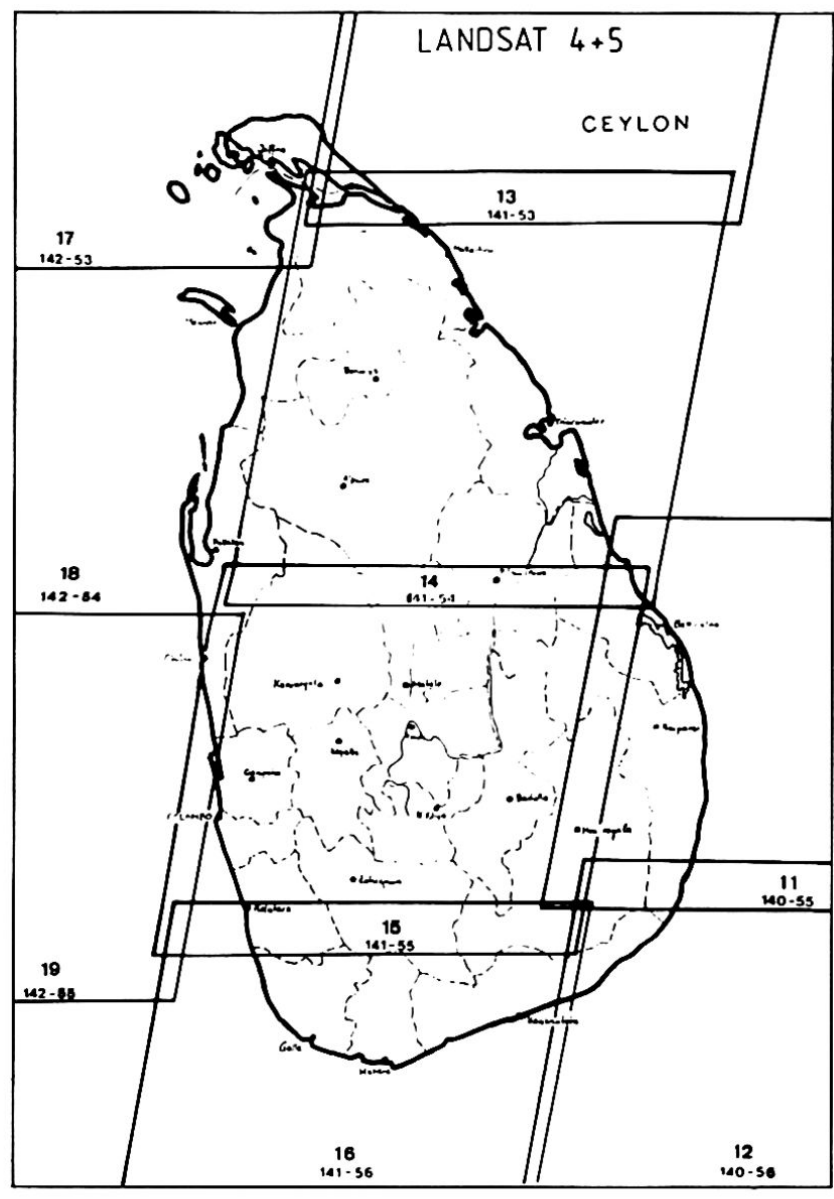

Abb. 1 Satellitenbildbedeckung Sri Lankas
sat-Bilder von den Stationen in Hyderabad, Indien, oder Bangkok, Thailand, bezogen werden (Abb. 2).

Im Projekt geht es jedoch nicht darum, Luftbildinterpretation durch die Auswertung von Satellitendaten zu ersetzen, im Gegenteil: die Pilotphase zeigte deutlich, daß eine kombinierte Verwendung verschiedener Fernerkundungstechniken für die Praxis beste Resultate liefert. Die gemeinsame Verwendung von Luft- und Satellitenbildern, ergänzt mit notwendigen Felduntersuchungen, wird als "Multistage-concept» bezeichnet.

Die grundsätzliche Frage nach dem Einsatz computerunterstützter Analyseverfahren oder konventioneller visueller Bildinterpretation wurde durch die Tatsache beantwortet, daß das Projekt kurzfristig benötigte Planungsdaten erheben soll. Der großräumige Einsatz digitaler Verfahren ist erst in Entwicklung begriffen und verlangt nach massiven technischen, finanziellen und personellen Investitionen. Grundbegriffe der analogen, visuellen Interpretation sind jedoch in Sri Lanka bereits vorhanden, so daß ein Aufbauen auf diesem Ansatz angepaßter und erfolgversprechender erschien (SSRSP 1979, NANAYAKKARA 1983).

\subsection{Zum thematischen Konzept}

Die Pilotphase begann mit einer gezielten sektoralen Fragestellung. Die traditionell verzögerten Reisernteschätzungen in den großen Bewässerungsgebieten sollten mit dem Einsatz von Satellitenbildern verbes-

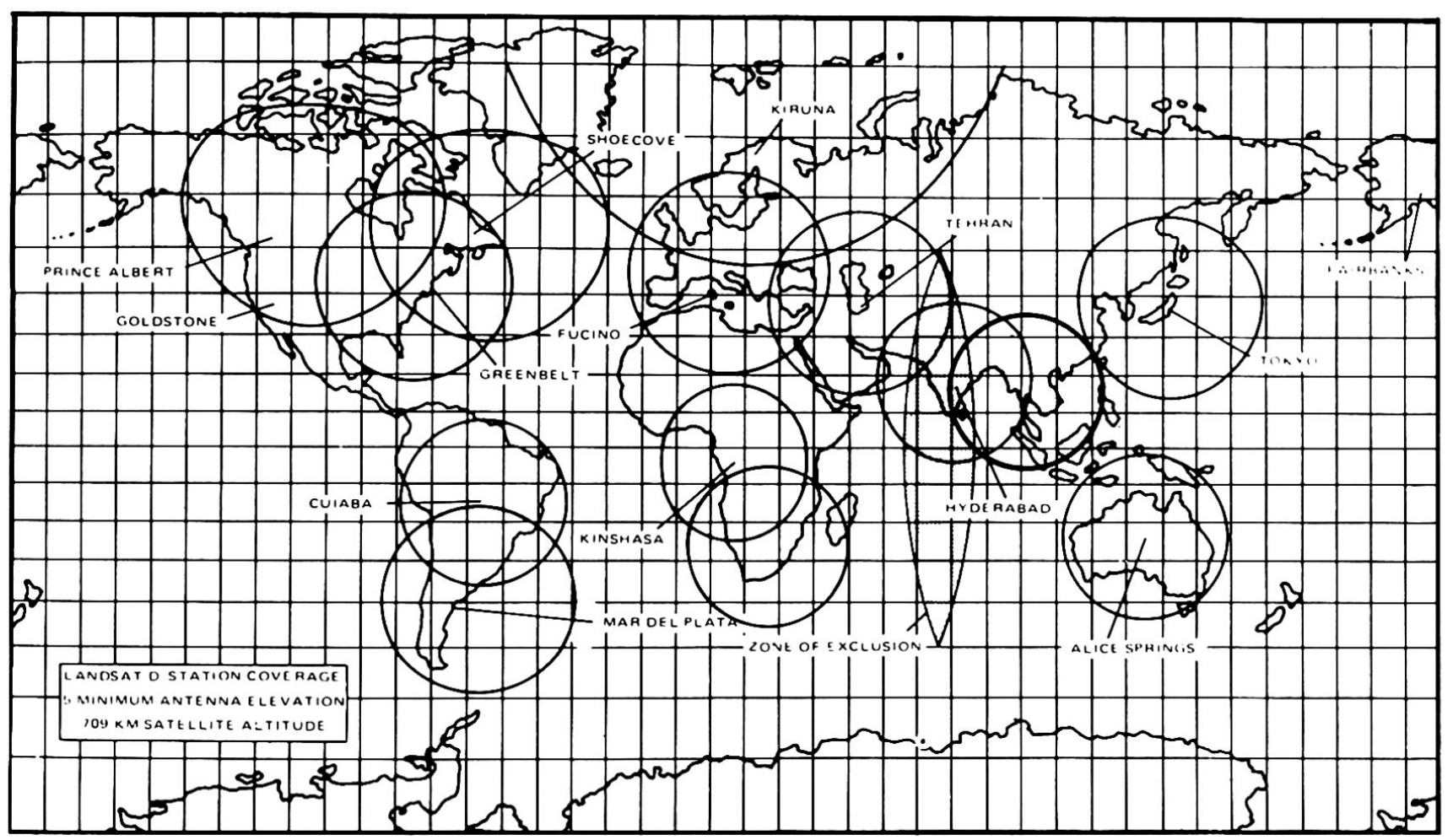

Abb. 2 Lage und Empfangsbereich der Boden-Stationen für Landsat 
sert werden und vor allem rascher zur Verfügung stehen. Die Untersuchungen zeigten jedoch, daß für diese Fragestellung auch die gesamte für Reiskultur zur Verfügung stehende Anbaufläche wie auch andere Landnutzungskategorien berücksichtigt werden müssen.

Es drängte sich deshalb auf, den von weiteren Amtsstellen gemeldeten Datenbedürfnissen (etwa Forstamt und Stadtplanung) entgegenzukommen und die verschieden gelagerten Ansprüche in einem integralen Konzept zu verschmelzen. Dieses Konzept kann als «Benchmark Mapping-Monitoring Concept» bezeichnet werden (GEISER et al., 1982). Es beruht auf einer Gesamterhebung der bestehenden Nutzung mit einer nachfolgenden sektoralen Nachführung oder Detailanalyse.

"Benchmark Mapping»: Eine landesweite, detaillierte Erhebung der heutigen Nutzung bildet das Rückgrat des Konzeptes. Diese kann nicht mit Satellitenbildern durchgeführt werden, sondern erfolgt aufgrund neuer Luftaufnahmen. Die Kartierung geschieht flächenbedeckend (beim vergleichbaren Projekt der Arealstatistik in der Schweiz wird ein modernes Stichprobenverfahren verwendet; TRACHSLER/KÖLBL, 1978). Die resultierenden Kartenblätter im Maßstab 1:100000 sind distriktweise unterteilt und in Farbe gedruckt. "Monitoring»: Die im obigen Arbeitsschritt entstandenen Karten zeigen den Istzustand der Nutzung. Die aufgrund der Nutzungsdynamik notwendige Nachführung kann aus technischen und finanziellen Gründen nicht mit Luftbildern geschehen. Deshalb wurde hierzu ein Verfahren entwickelt, das die Verwendung von Satellitenbildern erlaubt: die Grundidee besteht darin, daß die obige Basiskarte regelmäßig mit neuen Satellitenbildern verglichen wird, um so Veränderungen festzustellen. Da von Planerseite verschiedene Ansprüche an eine Nachführung gestellt werden, wird diese sektoral behandelt.

Neben diesen thematischen Hauptaktivitäten, mit denen bereits ein breites Spektrum von Datenbedürfnissen koordiniert abgedeckt werden kann, werden vermehrt auch spezialisierte Untersuchungen unterstützt.

\subsection{Zum organisatorischen Konzept}

Rückgrat der Projektaktivitäten ist der Aufbau und die Ausrüstung eines Fernerkundungszentrums am bestehenden Survey Department in Colombo, Sri Lanka. Das Survey Department verfügt bereits über modern ausgerüstete Abteilungen in Kartographie, Photogrammetrie, über Photolabors und eine Druckerei, welche vom Zentrum mitbenutzt werden können. Zudem läßt sich hier auch die wichtige Koordination von topographischer und thematischer Kartierung effektiver durchführen.

Das Zentrum, das sogenannte "Centre for Remote Sensing», soll neben der Durchführung von konkreten
Datenerhebungen auch als Koordinationsstelle für Fernerkundungsaktivitäten in Sri Lanka agieren und auswärtige Interessenten in der Benützung von solchen Methoden beraten.

Der Ausbildung lokaler Fachkräfte kommt besondere Bedeutung zu, da die begonnenen Arbeiten kontinuierlich weiterzuführen sind und der Schweizer Projektbeitrag lediglich als eine Starthilfe anzusehen ist. Dem Personal soll das notwendige Fachwissen vermittelt werden, um fernerkundungsgestützte $\mathrm{Ar}$ beiten von der Planung über die Durchführung bis zum Schlußprodukt zu ermöglichen.

Das für die Untersuchungen notwendige thematische, meist naturwissenschaftliche Fachwissen ist am eher technisch orientierten Survey Department nur zum Teil vorhanden. Deshalb wurde ein "User-concept" entwickelt, in welchem thematische Spezialisten aus der Verwaltung oder von Hochschulen mit den Fernerkundungsfachleuten des Zentrums zusammenarbeiten. Dadurch kann gleichzeitig ein Beitrag zum Transfer der erhobenen Informationen in die für die Planung zuständige Behörde geleistet werden.

\section{Einige A spekte der Projekt-Umsetzung}

\subsection{Das "Centre for Remote Sensing"}

Die zu Beginn der Projektaktivitäten räumlich wie personell kleine thematische Kartierungsabteilung wurde im Laufe der letzten Jahre schrittweise ausgebaut und zeigt sich heute in der Form eines Großraumbüros. Die nun als Fernerkundungszentrum, "Centre for Remote Sensing», bezeichnete Stelle bildet einen Teil des Survey Departments und untersteht einem «Superintendant of Surveys». Während das Zentrum vor allem im Bereich von Bildinterpretation sowie Beratung, Planung und Koordination von Fernerkundungsarbeiten tätig ist, sind Bereitstellung gewisser Fernerkundungsdaten wie Luftbilder und die weitere Verarbeitung bis zum professionellen Endprodukt anderen technischen Abteilungen übertragen. Entsprechend dieser Betriebsstruktur ist das Zentrum zusammen mit den unterstützenden technischen Abteilungen einem "Deputy Surveyor General» unterstellt. Das Survey Department, welches vom «Surveyor General» geleitet wird, untersteht dem «Ministry of Lands and Land Developments.

Die technische Ausrüstung des Zentrums wurde dem vorgesehenen methodischen Ansatz angepaßt und besteht vor allem aus Instrumenten zur Unterstützung der visuellen analogen Bildinterpretation. Hierzu gehören etwa Spiegelstereoskope, ein Zoom-Stereoskop mit Diskussionstubus (v. a. für die Ausbildung), ein hochvergrößerndes Zoom-Binokular, ein analoges Instrument zur Bildinterpretation und verschiedene z. T. elektronische Flächenmeßgeräte. Zur Durchführung der Feldarbeit stehen Fahrzeuge zur Verfügung. 


\subsection{Die Ausbildung des Personals}

Zwei bereits am Survey Department arbeitende erfahrene Luftbildinterpreten formten den Kern der Mitarbeiter. Das Spektrum der Projektarbeiten verlangte jedoch nach der Rekrutierung von neuem Personal. Dies geschah auch verschiedentlich im Verlaufe der bisherigen Projektphasen, zum Teil verbunden mit dem Problem der Fluktuation, vorab dem Abwandern von Fachkräften etwa in die Ölstaaten (Brain Drain). Heute (November 1984) arbeiten am Zentrum insgesamt 20 Leute: der einheimische Leiter und sein Stellvertreter, 9 Interpreten, 5 Zeichner und 4 Administratoren. Hinzu kommen regelmäBig zwei bis vier Leute aus den Benützer-Organisationen.

Die Ausbildung der Leute, die bei ihrer Rekrutierung noch nie ein Luftbild gesehen hatten, wurde zu einer zentralen Aufgabe. Als Grundsatz wurde diese Ausbildung möglichst im Rahmen der konkreten Arbeit ("Non the job») durchgeführt: Nach einer etwa vierwöchigen Einführung begann bereits die direkte Mitarbeit an der Landnutzungs-Kartierung, womit eine rasche und motivationsfördernde Integration in den operationellen Arbeitsablauf erreicht werden konnte. Als Nachteil mag die von den Beratern zusätzlich notwendige Kontrollarbeit genannt sein. Es ist klar, daß Neubeginner die an die operationelle Arbeit gestellten Anforderungen nicht gleich erfüllen, so daß eingehende Überprüfung und allfällige Korrekturen notwendig sind.

In der Zwischenzeit wurde das Ausbildungsprogramm strukturiert und auf einen fünf Jahre dauernden Kurs angelegt (HUMBEL 1983). Die so ausgebildeten Fachkräfte bedurften innerhalb der personal-administrativen Struktur des Survey Departments eine klare und ihren Fähigkeiten angepaßte Berufsbezeichnung; Mitte 1983 wurde deshalb offiziell die neue Bezeichnung «Remote Sensing Technician» eingeführt.

\subsection{Zusammenarbeit mit Datenbenützern}

Die im Rahmen des «User-Concept» vorgesehenen Aktivitäten haben sich in einem breiten Maße entwikkelt und spielen sich im wesentlichen auf zwei Ebenen ab. Eine große Zahl potentieller Benützer wird regelmäßig über die Aktivitäten und Kapazitäten des Zentrums informiert. Konkretisiert sich eine Fragestellung, so berät das Zentrum über möglichen Einsatz von Fernerkundungsdaten, stellt diese auch bereit (v.a. Luft- und Satellitenbilder) und führt das mit der eigentlichen Problemlösung betraute Personal, das von der Datenbenützerseite gestellt wird, in die Bildinterpretation ein. Zu diesem Zweck werden von Zeit zu Zeit drei- bis vierwöchige Einführungskurse in Fernerkundung organisiert.

Mit bislang sechs Organisationen hat sich eine institutionalisierte Zusammenarbeit ergeben: dem Forstamt (Forest Department), dem Agrarzensusamt
(Department of Census and Statistics), der Teeforschungsbehörde (Tea Research Institute), der Stadtplanung (Urban Development Authority), der Behörde für Meeresressourcen-Forschung (National Aquatic Resources Agency), und der für archäologische Ausgrabungen zuständigen Stelle (Central Cultural Fund). Mitarbeiter dieser Stellen arbeiten intensiv am Zentrum, teilweise an der Landnutzungskartierung, teilweise an eigenen Untersuchungen, und werden auch vertiefter in Fernerkundung ausgebildet. Drei der sechs Organisationen wurden beim Aufbau eigener, kleiner Fernerkundungsabteilungen unterstützt, die nun autonom arbeiten, weiterhin aber eng mit dem Zentrum in Kontakt stehen, sei es zur Benützung spezifischer oder teurer Instrumente oder für komplexere Untersuchungen. Weitere Amtsstellen sind an ähnlichen dezentralen Kartierungsabteilungen interessiert (Geologisches Landesamt, Behörde für Meeresressourcen-Planung, Kautschuk-Forschungsinstitut).

\subsection{Landnutzungskartierung (Benchmark Mapping)}

Neben der Ausbildung und Beratung bildet die konkrete Kartierung der heutigen Landnutzung den arbeitsintensivsten Teil des Projektes. Die im Laufe der Testphase verfeinerte Kartierungsweise wird heute operationell angewandt, und verschiedene Distriktkarten sind bereits in Farbe gedruckt (Abb.3). Das technische Vorgehen kann folgendermaßen zusammengefaßt werden (Abb. 4):

Feldarbeit: Eine Gruppe von zwei bis drei Interpreten bearbeitet einen Distrikt. Vor Beginn der Kartierung besucht die Gruppe den Distrikt, studiert die lokale Landnutzung (Reconaissance Survey) und datiert gleichzeitig die wichtigsten Elemente der vorhandenen topographischen Karte auf (Straßen, Ortsnamen), so $\mathrm{da} \beta$ diese als Basis in die Landnutzungskarte eingegliedert werden kann.

Bildinterpretation: Ganz Sri Lanka wird mit dem Flugzeug des Survey Department (Cessna Golden Eagle 421-C) neu beflogen. Die Bilder in Maßstäben $1: 20000$ und $1: 50000$ dienen sowohl der Herstellung neuer topographischer Karten wie auch als Basis für die Interpretation der Landnutzung im entsprechenden Distrikt. Mittels eines Interpretationsschlüssels, dessen Erarbeitung sehr viel Zeit beanspruchte, werden die vielen hundert Luftaufnahmen ausgewertet. Die Klassifikation (Abb. 5) ist hierarchisch aufgebaut und erlaubt eine Erfassung der gesamten Flächenbedeckung in Hauptkategorien, die wiederum entsprechend den lokalen Verhältnissen oder dem Datenbedürfnis weiter unterteilt werden können.

Transfer auf Kartenbasis: Nach einer abschließenden Kontrolle der Bildinterpretationen im Felde müssen diese nun auf einer geometrisch korrekten Kartenbasis zusammengefügt werden. Die topographischen Karten im Maßstab 1:63360 (one inch to one mile) aus der Zeit der britischen Verwaltung sind meist 


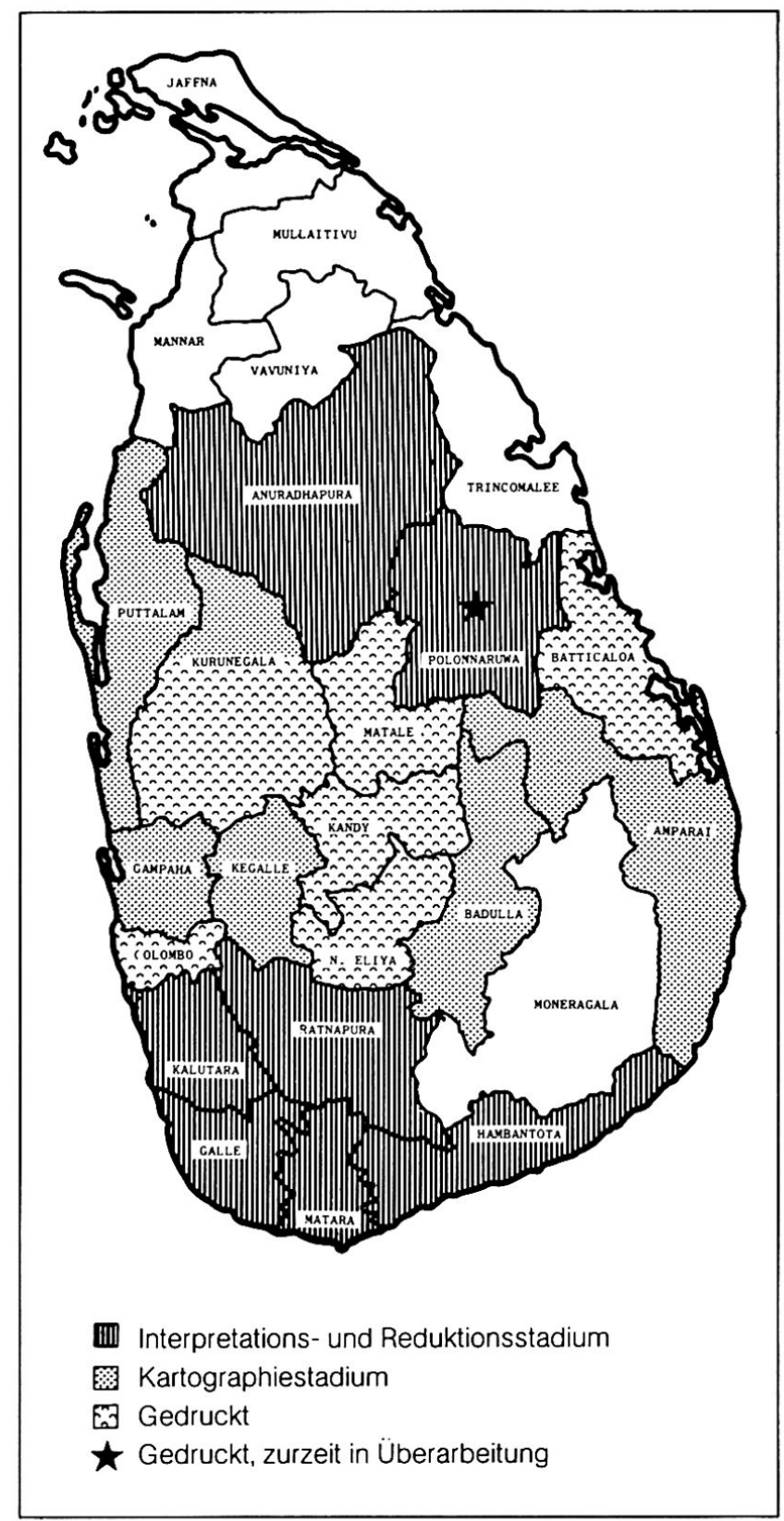

Abb. 3 Stand der Landnutzungskartierung Ende 1984

veraltet und können nur teilweise als Basis herangezogen werden. Deshalb wurde ein Verfahren entwickelt, welches eine Kombination von bestehender topographischer Karte und Satellitenbild ergibt. Wolkenfreie Satellitenbilder im Originalmaßstab 1:1 Million werden geometrisch korrigiert, bildkontrastgesteigert, auf den Maßstab 1:100000 farbig vergrößert und gemäß der Gliederung der topographischen Karte unterteilt. Ausgehend von digitalen Satellitendaten entsteht so ein landesbedeckender Satz von qualitativ hochstehenden Bildern. Für die Nachführung wird mit finanziell erheblich günstigeren photographischen Produkten gearbeitet. Die digitale Aufbereitung geschieht am Geographischen Institut der Universität Zürich in Zusammenarbeit mit dem Institut für
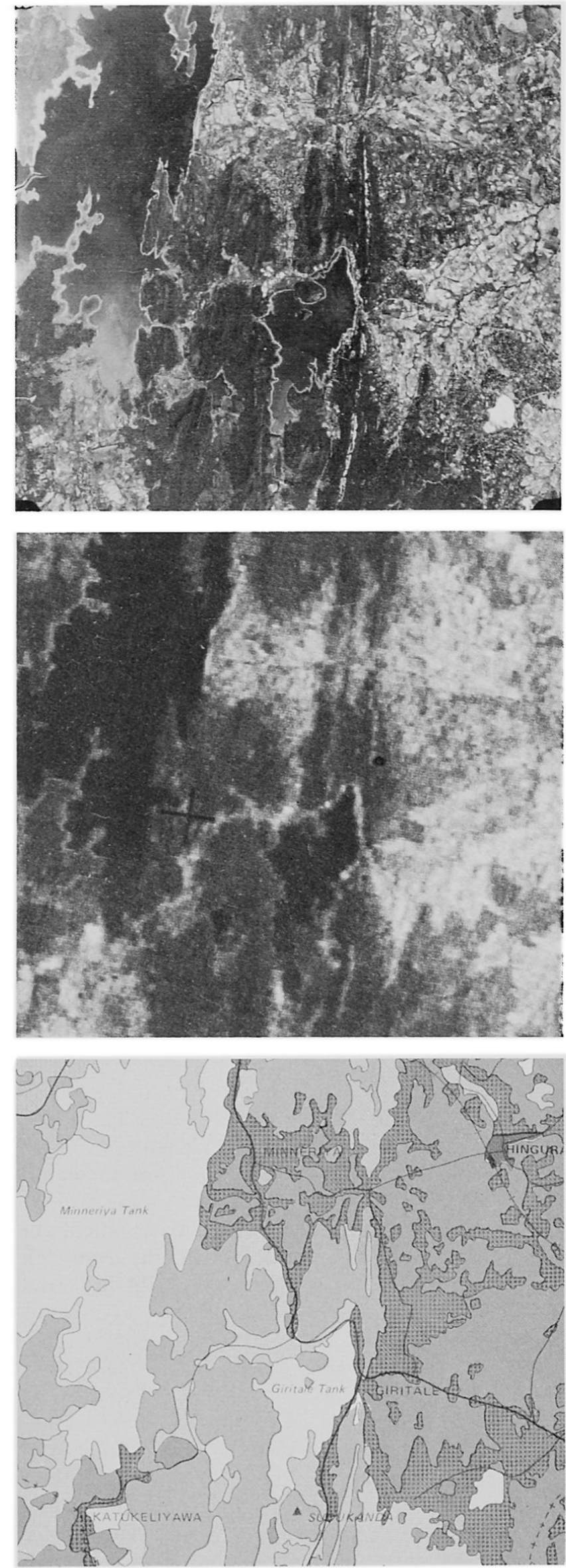

Abb. 4 Luftbild (oben), Satellitenbild (Mitte) und daraus hergestellte Karte (unten; ca. $1: 200$ 000) 


\begin{tabular}{|c|c|c|}
\hline URBAN LAND & \multicolumn{2}{|c|}{ BUILT.UP LAND } \\
\hline \multicolumn{3}{|c|}{ AGRICULTURAL LAND } \\
\hline & \multicolumn{2}{|l|}{ HOMESTEADS } \\
\hline & TREE AND OTHER & TEA \\
\hline & \multirow[t]{3}{*}{ PERENNIAL CROPS } & RUBBER \\
\hline & & COCONUT \\
\hline & & OTHER PERENNIAL CROPS \\
\hline & \multirow[t]{3}{*}{ CROPLAND } & PADDY \\
\hline & & SPARECELY USED CROPLD \\
\hline & & OTHER CROPLAND \\
\hline \multirow[t]{4}{*}{ FOREST LAND } & \multirow[t]{3}{*}{ NATURAL FOREST } & DENSE FOREST \\
\hline & & OPEN FOREST \\
\hline & & MANGROVES \\
\hline & \multicolumn{2}{|c|}{ FOREST PLANTATIONS } \\
\hline \multirow[t]{2}{*}{ RANGELAND } & \multicolumn{2}{|l|}{ SCRUBLAND } \\
\hline & \multicolumn{2}{|l|}{ GRASSLAND } \\
\hline \multicolumn{3}{|c|}{ WATER (TANKS, RIVERS, LAGOONS, SEA) } \\
\hline \multicolumn{3}{|c|}{ BARREN LAND (MAINLY ROCK, SAND) } \\
\hline
\end{tabular}

Abb. 5 Klassifikationsschlüssel der Landnutzung

Kommunikationstechnik der ETH. Die topographische Karte ihrerseits wird auf Transparentfilm im Maßstab 1:100000 reduziert, generalisiert und paßgenau dem entsprechenden Ausschnitt des Satellitenbildes überlegt. Die schließlich resultierende Kombination von Karte und Satellitenbild besitzt einen Detailreichtum, der den optischen Transfer der Luftbildinterpretation auf diese kartographische Basis möglich macht. Gewisse Interpretationen können auch direkt auf dem Satellitenbild durchgeführt werden (Wasser, z. T. Wald).

Kartographie: Die kompilierten Interpretationen werden nun distriktweise zusammengestellt und kartographisch bearbeitet. Die für einen Farbdruck notwendigen Schritte beinhalten etwa das Gravieren der Grenzlinien auf speziellen Folien, das Herstellen von Farbauszügen, das Zeichnen der Basiskarte und Zusatzinformationen usw. Die Flächenwerte jeder Kategorie werden pro Subdistrikt mit einem elektrooptischen Flächenmeßgerät ermittelt und ebenfalls auf die Karte gedruckt. Der Farbdruck geschieht am Survey Department in sechs Farben, die zum Teil aufgerastert oder mit Strukturrastern überlegt sind (Abb.6).

\subsection{Die Sektorale Nachführung (Monitoring)}

Die unter 2.3 kurz skizzierte Methode beruht auf der paßgenauen Überlagerung von neuen Satellitenbildern mit der Landnutzungskarte, welche gleichsam als Basis- oder Referenzinventur gilt. Da die Abgrenzung einzelner Nutzungskategorien auf der Landnutzungskarte gegeben ist, muß diese auf dem Satellitenbild nicht wiederholt werden. Von der Karte werden für einzelne Landnutzungsklassen Masken hergestellt, welche das umliegende Gebiet abdecken und eine ungestörte Analyse von Farb- oder Grautonunterschieden der entsprechenden Landfläche auf dem Satellitenbild ermöglichen. Die Überlagerung geschieht mittels einfacher optischer Projektion (Abb. 7).

Das Verfahren wird bereits zur regelmäßigen Beobachtung von Waldflächenveränderungen eingesetzt (Abb. 8).

\subsection{Weitere Arbeiten}

Neben den geschilderten Hauptaufgaben befaßt sich das Zentrum mit weiteren operationellen oder in Entwicklung begriffenen Programmen, von denen einzelne kurz erwähnt sein sollen:

Reisflächenuntersuchungen: Mit der Technik der sektoralen Nachführung (Maskierung) kann die jeweils saisonal angebaute Reisfläche auf einem Satellitenbild, das rund einen Monat vor der Ernte aufgenommen wurde, kartiert werden. Das Verfahren ist in Entwicklung begriffen, und der Einbezug der Flächenzahlen als Parameter in einem Reisertragsprognosemodell wurde überprüft (TSCHANNEN 1982). Erhebliche Abweichungen der Flächenzahlen wie der Prognosen im Vergleich zu amtlichen Agrarstatistiken erforderten eine Spezialstudie zur Genauigkeitsüberprüfung (GRIesbaum 1983). Da sich ähnliche Probleme im Vergleich anderer Flächenstatistiken (z.B. Plantagengebiete) stellen, ist ein Angehen und Lösen dieses Problemkreises sehr notwendig.

Städtische Landnutzung: In enger Zusammenarbeit mit der Stadtplanungsbehörde entstehen Spezialkarten urbaner Räume, die überwiegend auf einer Interpretation von Luftaufnahmen mit begleitender Feldbegehung beruhen (sOMMER et al. 1982).

Küstenschutz: In Zusammenarbeit mit der zuständigen Amtsstelle entstanden verschiedene Detailaufnahmen zur Sedimentation in Mündungsbereichen und zu Mangrovenbeständen.

Waldbestand: Auf Anfrage des zuständigen Ministeriums entstand die erste landesweite Karte der noch bestehenden Waldfläche aufgrund von Satellitenbildern und Feldbegehung. Die Karte im Maßstab 1:500 000 wurde 1981 als Farbdruck herausgegeben (Abb.9) und wird gegenwärtig nachgeführt.

Vegetationsschäden: Schädlingsbefall in der Plantagenwirtschaft wird zunehmend zum Problem. In Zusammenarbeit mit den Forschungsstellen für Tee und Kautschuk hat eine Untersuchung zur möglichen Verwendung von Falschfarbenfilmen für die Früherkennung des Schadenbefalls begonnen.

Archäologie: In Zusammenarbeit mit dem Archäologischen Amt sind Falschfarbenfilme erfolgreich zur Lokalisierung vergrabener Strukturen eingesetzt worden (GELBERT et al. 1984). Das Verfahren soll nun weiter Verwendung finden. 


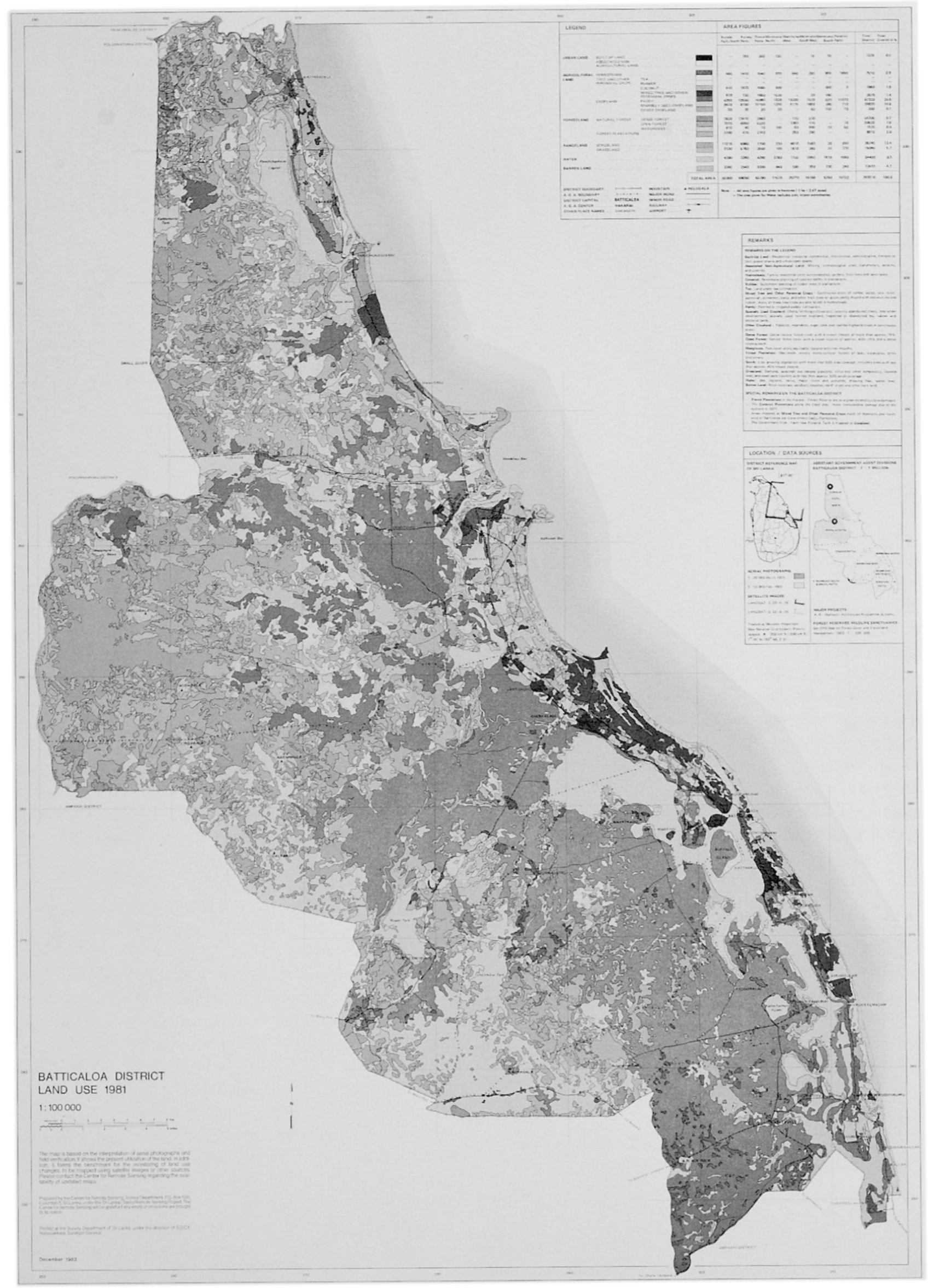

Abb. 6 Landnutzungskarte des Distrikts Batticaloa, 1981 (Originalmaßstab 1:100 000, hier ca. 1:500 000) 


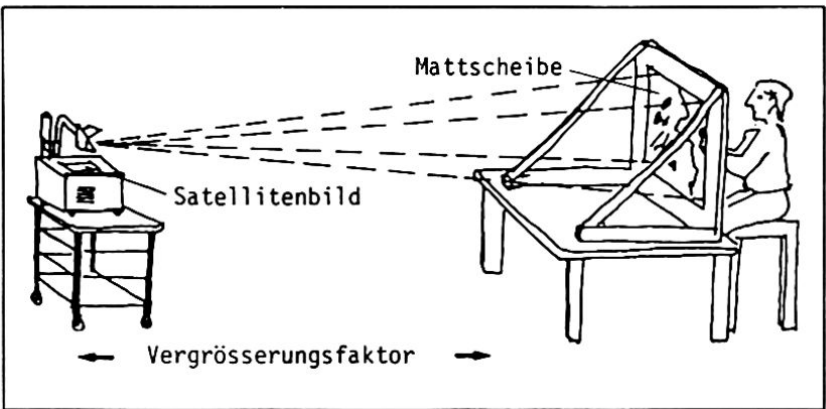

Abb. 7 Nachführen der Karte mit Hilfe von Satellitenbildern

\section{Zum Projekt - Nachfeld}

Die im Rahmen des Projektes aufgearbeiteten Informationen richten sich in erster Linie an Planer und Entscheidungsträger innerhalb der Administration Sri Lankas. Dazu gehören Offizielle von sektoralen Ministerien, welche Einzelprojekte oder inter-ministeriale Maßnahmen aufgrund rezenter Informationen auszuführen haben, Fachleute in einzelnen Departementen usw. Als zweite Zielgruppe ist die Regionalverwaltung zu nennen, welche viele Projekte in ihrer Ausführung direkt betreut (deshalb die Landnutzungskarten auf Distriktbasis). Als dritte Gruppe sind die zahlreichen Forschungs- und Lehrstätten des Landes angesprochen, und schließlich ist auch die Vielzahl von nichtstaatlichen Organisationen und Interessengruppen (etwa im Bereich ländlicher Entwicklung oder Umwelt) auf umfassende und zuverlässige Informationen angewiesen.

In diesem Rahmen stellt sich die Frage, wieweit das Projekt als Daten- und Informationshersteller dieses Material in die konkrete Anwendung und Planung begleiten soll oder muß. Für den Einsatz in Sri Lanka hat sich gezeigt, daß eine übergreifende Zusammenarbeit zwischen dem Zentrum als Informationsbeschaffer mit den Benützern (Planer, Wissenschafter usw.) einen wechselwirkenden Proze $\beta$ in Gang bringt, der eine effiziente Ausrichtung der Arbeit auf die Bedürfnisse der Praxis ermöglicht.

Für die weitere Begleitung der Informationen in den Planungs- und Anwendungsbereichen sind verschiedene Maßnahmen in Diskussion, z.B. Information durch die Medien, Kurzzeitseminarien und Workshops in den Distrikten usw.

\section{Versuch einer Zwischenbilanz}

Die im vorliegenden Artikel nur knapp geschilderten Projektaktivitäten sind in vollem Gange. Eine Vielzahl konkreter Resultate wurde bereits erreicht, während in anderen Bereichen an Entwicklung und Konkretisierung gearbeitet wird; der dauernde Lernprozeß geht
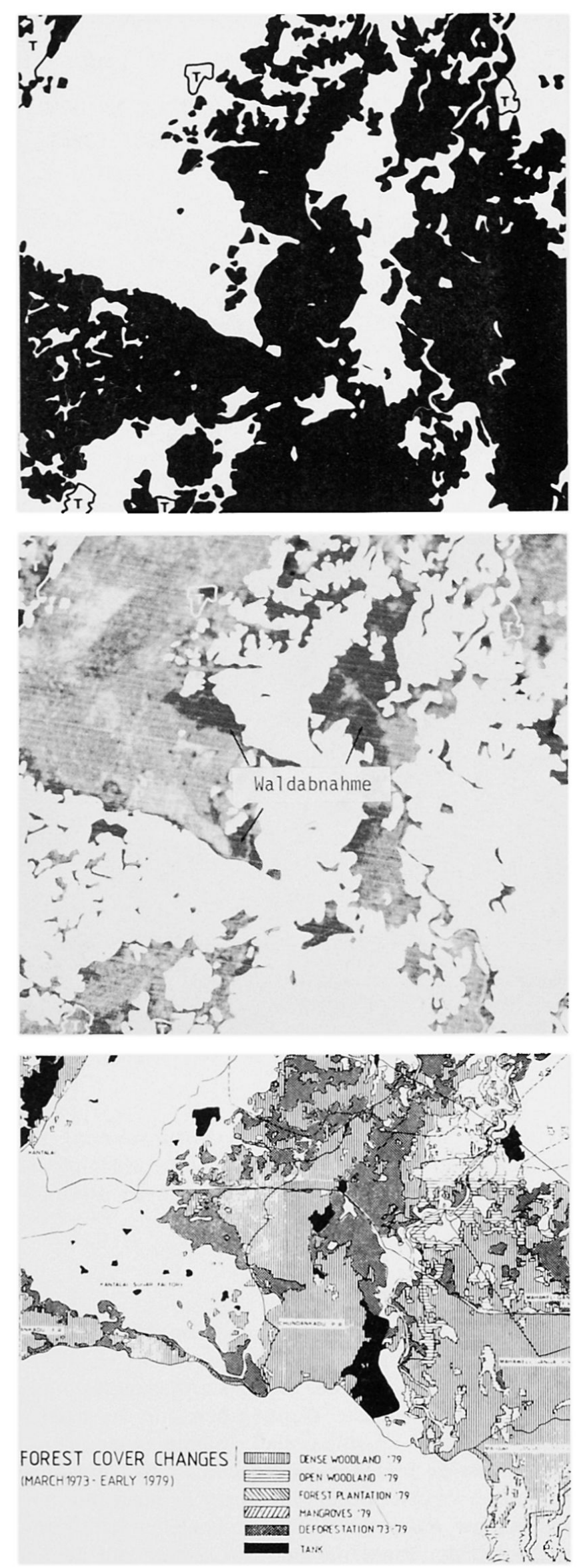

Abb. 8 Waldmaske 1979 (oben) überlegt auf ein Satellitenbild von 1973 zeigt die Waldveränderung (unten); (Maßstab ca. $1: 200000$ ) 


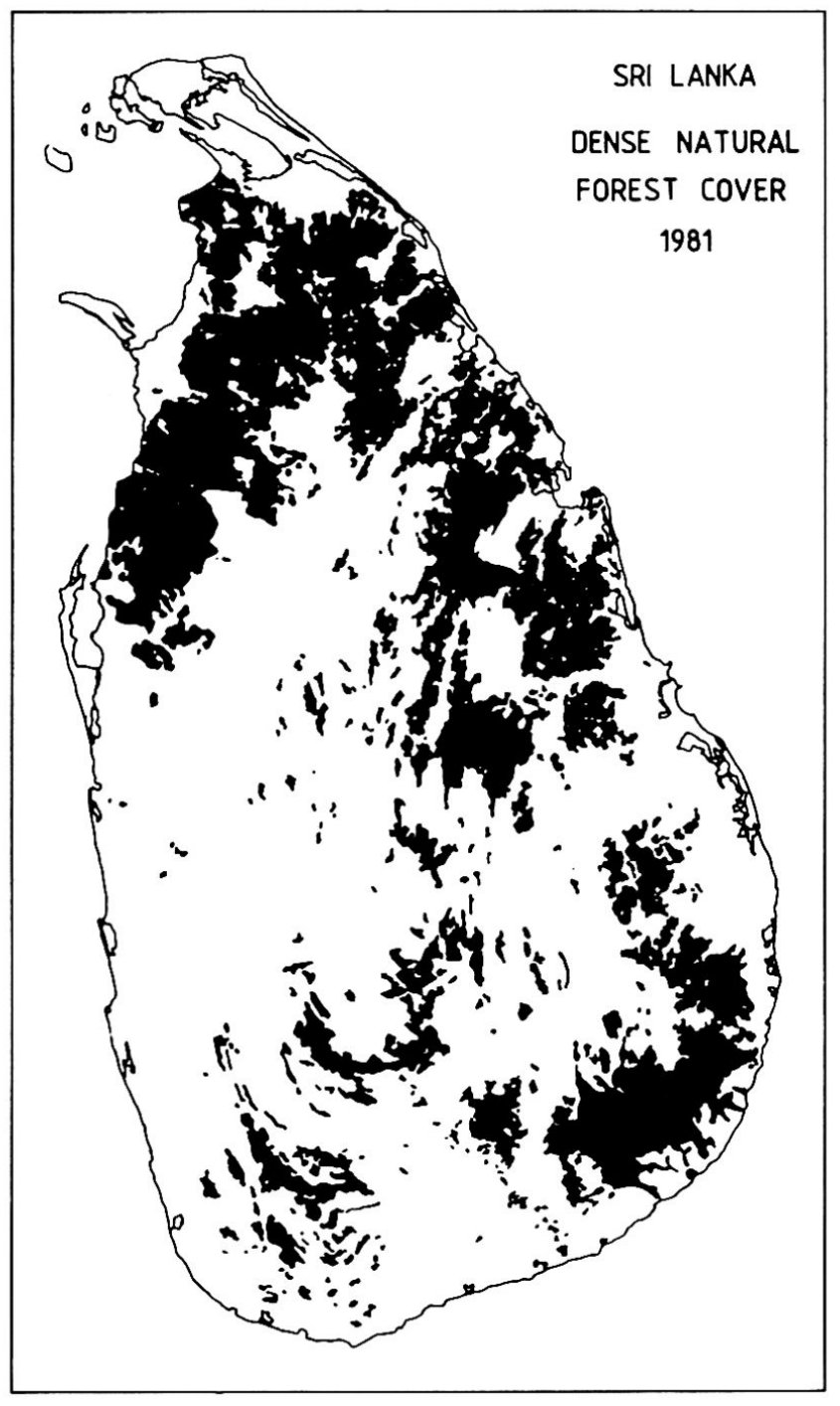

Abb. 9 Waldbestandeskarte 1981 (dichter, natürlicher Wald, Originalmaßstab $1: 500$ 000, 1981)

weiter. Es soll im folgenden versucht werden, die verschiedenen Arbeitsbereiche im Sinne einer provisorischen Zwischenbilanz stichwortartig zu diskutieren.

\subsection{Thematischer Bereich}

Das Projekt ist überzeugt, mit den von ihm bereitgestellten Daten und Informationen einen wichtigen Beitrag zur Behebung des Defizits an Grundlagendaten für verschiedenste Planungsbereiche zu liefern (Agrarplanung, Forstwirtschaft, Stadtplanung, Umweltdiskussion und Ressourcenschutz usw.). Im Verlauf dieser Arbeit entsteht ein enger Kontakt mit den zuständigen Planungsstellen mit begleitendem Kennenlernen der Probleme und Strukturen, mit denen eben diese Planung zu arbeiten hat. Die hier anstehenden Fragen etwa der Planungsansätze und -ziele, der Koordination usw., berühren auch die Tätigkeit des Datenbeschaffenden. Wichtig ist, daß die ceylonesische Regierung koordinierende Bemühungen in der Planung unternimmt; daß dies geschieht, zeigt z. B. die Bildung einer Landplanungsstelle (Land Use Policy Planning Division), welche eine Straffung und Koordination der Planungsabläufe zum Ziele hat. Sie wurde deshalb zu einem wichtigen Partner des Projektes.

\subsection{Technischer Bereich}

Das Konzept der integralen Landnutzungskartierung und der sektoralen Nachführung hat sich als sinnvoller Ansatz erwiesen, welcher eine Koordination und Optimierung der Datenerhebung ermöglicht und zum Teil sogar zu einem Dialog unter den verschiedenen Benützern führt.

Die Diskussion um Fernerkundung und «angepaßte Technologie» ist sehr vielschichtig und komplex. Tatsache ist, daß der im vorliegenden Projekt gewählte Ansatz eine effiziente Erfassung wichtiger und relevanter Informationen zuläßt, die schwerlich auf anderem Wege zuverlässig zu erheben wären. Den ceylonesischen Mitarbeitern stellen sich bei der Übernahme der zum Teil neuen Methoden kaum Probleme. Die Partnerorganisation ist aber auch interessiert, nicht nur traditionell bewährte Technologien zu übernehmen. Sie möchte auch Starthilfe für die digitale Bildverarbeitung bekommen, um so den Anschluß an die Entwicklung im Norden nicht zu verpassen.

Die Lieferung von Satellitenbildern durch die indische und die thailändische Station gestaltete sich bislang recht schwerfällig und führte teilweise zu mehrmonatigen Lieferverzögerungen. Es zeichnet sich jedoch eine Verbesserung dieser Situation ab. Daneben wird auch an alternative Möglichkeiten der regelmäßigen und großflächigen Erfassung gedacht, wie etwa Weitwinkel-Luftaufnahmen aus großer Höhe.

\subsection{Personeller Bereich}

Die ceylonesischen Fachkräfte haben sich in den ersten Jahren des Projektes ein sehr gutes Fachwissen angeeignet, und deshalb stellen sich kaum Probleme bei der technischen Umsetzung der Arbeiten. Ein schwieriger Aspekt ist jedoch die Fluktuation des Personals. Dieses häufig als «Brain Drain» bezeichnete Phänomen betrifft nicht nur das Fernerkundungszentrum, sondern den ganzen staatlichen Sektor in Sri Lanka wie auch viele andere Länder der Dritten Welt. In den letzten Jahren sind die Löhne im öffentlichen Sektor weit hinter denjenigen der in Sri Lanka stark geförderten Privatwirtschaft zurückgefallen. So werden in der Privatwirtschaft auf vergleichbarer Ebene rund die doppelten, im Ausland, hier vor allem in den Erdölstaaten des Mittleren Ostens, die acht- bis zwanzigfachen Löhne angeboten. $\mathrm{Da} \beta$ in einem 
solchen Umfeld kaum an Idealismus appelliert werden kann, muß sicherlich verstanden werden. Die sehr hohen Lebenshaltungskosten machen eine Staatsstelle wenig attraktiv. Davon betroffen sind vor allem die Bereiche der Leitung und des Management. Das Projekt unternimmt auch hier Anstrengungen. Es wurde versucht, Zusatzzahlungen für besondere Arbeitsleistungen zu erreichen, was allerdings angesichts der anderen Staatsstellen mit gleichen Problemen wenig aussichtsreich ist. Auf der pragmatischen Ebene wird das Problem mit einer kontinuierlichen Neurekrutierung und Ausbildung angegangen. So übernehmen die schon ausgebildeten Leute auch selber die Rolle des Ausbildners. Dadurch wird versucht, eine Kontinuität der Ausbildung zu erreichen. Es genügt nicht, eine Gruppe von Fachkräften etwa durch Schweizer Berater auszubilden; der Ausbildungsproze $\beta$ selber muß formalisiert und etabliert werden. In diesem Zusammenhang sucht das Zentrum auch die Zusammenarbeit mit den ceylonesischen Hochschulen. Diese Anstrengungen konnten die Personallage auf der technischen Ebene, wo auch der Hauptteil der Mitarbeiter tätig ist, weitgehend stabilisieren. Für die Leitungsebene des Zentrums wird an einer langfristig tragfähigen Lösung gearbeitet.

\subsection{Organisatorischer Bereich}

Das Zentrum für Fernerkundung in Colombo ist eine an sich kleine Amtsstelle, die von ihrem räumlichen wie finanziellen Umfang kaum mit ähnlichen Institutionen etwa in Bangla Desh, Indien, oder anderen Ländern der Dritten Welt verglichen werden kann. Dafür mag sie für sich in Anspruch nehmen, daß die gezielt operationelle Ausrichtung ihrer Aktivitäten einen beträchtlichen Umfang von Informationsbeschaffung ermöglichte. Hierfür war sicherlich auch die Integration der Stelle in eine bestehende Institution hilfreich, da dadurch auf bestehendes Fachwissen und Erfahrung in verschiedenen Teilbereichen aufgebaut werden konnte.

Als gewisser Nachteil einer solchen Integration sollte die Einbettung in den bestehenden Verwaltungsaufbau genannt sein. Die ceylonesische Bürokratie, welche in ihren Strukturen wie etwa Hierarchie, streng horizontale Berufsstruktur, Aufstiegschancen, Verantwortlichkeit, Innovationsfähigkeit usw. immer noch auf den in der Kolonialzeit festgelegten Mustern beruht, findet es in der heutigen dynamischen und von vielen Ansprüchen gekennzeichneten Zeit sehr schwierig, sich zu behaupten. Davon ist auch das Zentrum etwa im Bereich der Planung und Koordination von Arbeitsabläufen betroffen. Es muß sich deshalb mit den vielfältigsten Problemen dieses Themenkreises befassen.

Das Projekt versucht in Zusammenarbeit mit den ceylonesischen Stellen, hier eine bessere Flexibilität zu erreichen. Für die Zukunft ist der Ausbau und die Aufwertung des Zentrums zu einem nationalen
Zentrum (National Centre for Remote Sensing) geplant. Ein entsprechender Kabinettsbeschluss wurde von der ceylonesischen Regierung bereits gefaßt. Es ist zu hoffen, daß dadurch eine Verbesserung der Autonomie und die Optimierung des Arbeitsprozesses ermöglicht werden kann. Trotz dieser Probleme übt das Zentrum eine bedeutende Dienstleistungsfunktion für verschiedenste Planungsbereiche aus, die wohl kaum im Rahmen privatwirtschaftlicher Strukturen angeboten werden können.

\section{Literatur}

ACRS (1983): Proceedings of The Fourth Asian Conference on Remote Sensing (ACRS); November 10-15, 1983, Colombo, Sri Lanka.

GEISER, U. et al. (1982): Multistage Landuse Mapping and Monitoring in Sri Lanka; Proceedings of an EARSel-ESA Symposium, Igls, Austria, April 1982.

GEISER, U. et al. (1983): Monitoring Deforestation in Sri Lanka through visual Interpretation of Landsat Imagery; in: ACRS (1983).

GELBERT, M. et al. (1984): The Use of Infrared Photography in Archeology. The Example of the Archeological Site of Sigiriya, Sri Lanka; In: Proceedings of the Fifth ACRS, Kathmandu, Nepal.

GRIESBAUM, M. (1983): Genauigkeitsuntersuchung von Flächenerhebungen für ein Reisernteprognosemodell; Diplomarbeit Geographisches Institut Universität Zürich.

HAEFNER, H. (1975): Erderkundung aus dem Weltraum; Geographica Helvetica Nr. 3.

HUMBEL, R. (1983): A Praxis-oriented, integral Training Programme for Remote Sensing Technicians; in: ACRS (1983).

NANAYAKKARA, S.D.F.C. et al. (1983): A National Land Use Inventory for Sri Lanka; in ACRS (1983).

SOMMER, M. et al. (1982): Land Utilization Maps for the Colombo Metropolitan Region based on Remote Sensing Techniques; Development Planning Review, Vol. 1, Nos 1 \& 2.

SSRSP (1978): Rice Yield Forecasting with Landsat Imagery; Sri Lanka - Swiss Remote Sensing Project: SSRSP Report on the Pilot Project, Colombo, Zurich.

SSRSP (1979): Rice Yield Forecasting and Monitoring Land Use Changes using Satellite Images; Sri Lanka - Swiss Remote Sensing Project: A Primer, Zurich, Colombo.

SSRSP (1981): Report on the Testphase: Mapping of Land Use as a Benchmark for Monitoring of Forest Cover Changes and Paddyland; Sri Lanka - Swiss Remote Sensing Project, Colombo.

SSRSP (1981, 1982, 1983): Annual Reports, Colombo.

TRACHSLER, H. et al. (1978): Großräumige Landnutzungserhebungen mittels stichprobenweisen Auswertungen von Luftbildern; DISP Nr. 51, ORL Institut ETH., Zürich.

TSCHANNEN, M. (1982): Aufbau eines regionalen Reisertragsprognosemodells am Beispiel des Polonnaruwa-Distriktes, Sri Lanka; Diplomarbeit, Geographisches Institut, Universität Zürich. 